\title{
Riesz transforms associated with Schrödinger operators acting on weighted Hardy spaces
}

\author{
Hua Wang* \\ School of Mathematical Sciences, Peking University, Beijing 100871, China
}

\begin{abstract}
Let $L=-\Delta+V$ be a Schrödinger operator acting on $L^{2}\left(\mathbb{R}^{n}\right), n \geq 1$, where $V \not \equiv 0$ is a nonnegative locally integrable function on $\mathbb{R}^{n}$. In this article, we will introduce weighted Hardy spaces $H_{L}^{p}(w)$ associated with $L$ by means of the area integral function and study their atomic decomposition theory. We also show that the Riesz transform $\nabla L^{-1 / 2}$ associated with $L$ is bounded from our new space $H_{L}^{p}(w)$ to the classical weighted Hardy space $H^{p}(w)$ when $\frac{n}{n+1}<p<1$ and $w \in A_{1} \cap R H_{(2 / p)^{\prime}}$. MSC: 35J10; 42B20; 42B30

Keywords: Weighted Hardy spaces; Riesz transform; Schrödinger operator; atomic decomposition; $A_{p}$ weights
\end{abstract}

\section{Introduction}

Let $n \geq 1$ and $V$ be a nonnegative locally integrable function defined on $\mathbb{R}^{n}$, not identically zero. We define the form $\mathcal{Q}$ by

$$
\mathcal{Q}(u, v)=\int_{\mathbb{R}^{n}} \nabla u \cdot \nabla v d x+\int_{\mathbb{R}^{n}} V u v d x
$$

with domain $\mathcal{D}(\mathcal{Q})=\mathcal{V} \times \mathcal{V}$ where

$$
\mathcal{V}=\left\{u \in L^{2}\left(\mathbb{R}^{n}\right): \frac{\partial u}{\partial x_{k}} \in L^{2}\left(\mathbb{R}^{n}\right) \text { for } k=1, \ldots, n \text { and } \sqrt{V} u \in L^{2}\left(\mathbb{R}^{n}\right)\right\} .
$$

It is well known that this symmetric form is closed. Note also that it was shown by Simon [17] that this form coincides with the minimal closure of the form given by the same expression but defined on $C_{0}^{\infty}\left(\mathbb{R}^{n}\right)$ (the space of

*E-mail address: wanghua@pku.edu.cn. 
$C^{\infty}$ functions with compact supports). In other words, $C_{0}^{\infty}\left(\mathbb{R}^{n}\right)$ is a core of the form $\mathcal{Q}$.

Let us denote by $L$ the self-adjoint operator associated with $\mathcal{Q}$. The domain of $L$ is given by

$$
\mathcal{D}(L)=\left\{u \in \mathcal{D}(\mathcal{Q}): \exists v \in L^{2} \text { such that } \mathcal{Q}(u, \varphi)=\int_{\mathbb{R}^{n}} v \varphi d x, \forall \varphi \in \mathcal{D}(\mathcal{Q})\right\}
$$

Formally, we write $L=-\Delta+V$ as a Schrödinger operator with potential $V$. Let $\left\{e^{-t L}\right\}_{t>0}$ be the semigroup of linear operators generated by $-L$ and $p_{t}(x, y)$ be their kernels. Since $V$ is nonnegative, the Feynman-Kac formula implies that

$$
0 \leq p_{t}(x, y) \leq \frac{1}{(4 \pi t)^{n / 2}} e^{-\frac{|x-y|^{2}}{4 t}}
$$

for all $t>0$ and $x, y \in \mathbb{R}^{n}$.

The operator $\nabla L^{-1 / 2}$ is called the Riesz transform associated with $L$, which is defined by

$$
\nabla L^{-1 / 2}(f)(x)=\frac{1}{\sqrt{\pi}} \int_{0}^{\infty} \nabla e^{-t L}(f)(x) \frac{d t}{\sqrt{t}} .
$$

This operator is bounded on $L^{2}\left(\mathbb{R}^{n}\right)$ (see [11]). Moreover, it was proved in $[1,3]$ that by using the molecular decomposition of functions in the Hardy space $H_{L}^{1}\left(\mathbb{R}^{n}\right)$, the operator $\nabla L^{-1 / 2}$ is bounded from $H_{L}^{1}\left(\mathbb{R}^{n}\right)$ into $L^{1}\left(\mathbb{R}^{n}\right)$, and hence, by interpolation, is bounded on $L^{p}\left(\mathbb{R}^{n}\right)$ for all $1<p \leq 2$. Now assume that $V \in R H_{q}$ (Reverse Hölder class). In [15], Shen showed that $\nabla L^{-1 / 2}$ is a Calderón-Zygmund operator if $q \geq n$. When $\frac{n}{2} \leq q<n$, $\nabla L^{-1 / 2}$ is bounded on $L^{p}\left(\mathbb{R}^{n}\right)$ for $1<p \leq p_{0}$, where $1 / p_{0}=1 / q-1 / n$, and the above range of $p$ is optimal. For more information about the Hardy spaces $H_{L}^{p}\left(\mathbb{R}^{n}\right)$ associated with Schrödinger operators for $0<p \leq 1$, we refer the readers to $[4,5,6]$.

In [18], Song and Yan introduced the weighted Hardy spaces $H_{L}^{1}(w)$ associated to $L$ in terms of the area integral function and established their atomic decomposition theory. In the meantime, they also showed that the Riesz transform $\nabla L^{-1 / 2}$ is bounded on $L^{p}(w)$ for $1<p<2$, and bounded from $H_{L}^{1}(w)$ to the classical weighted Hardy space $H^{1}(w)$.

As a continuation of [18], the main purpose of this paper is to define the weighted Hardy spaces $H_{L}^{p}(w)$ associated to $L$ for $0<p<1$ and study their atomic characterizations. We also obtain that $\nabla L^{-1 / 2}$ is bounded from $H_{L}^{p}(w)$ to the classical weighted Hardy space $H^{p}(w)$ for $\frac{n}{n+1}<p<1$. Our main result is stated as follows. 
Theorem 1.1. Suppose that $L=-\Delta+V$. Let $\frac{n}{n+1}<p<1$ and $w \in$ $A_{1} \cap R H_{(2 / p)^{\prime}}$. Then the operator $\nabla L^{-1 / 2}$ is bounded from $H_{L}^{p}(w)$ to the classical weighted Hardy space $H^{p}(w)$.

It is worth pointing out that when $L=-\Delta$ is the Laplace operator on $\mathbb{R}^{n}$, then the space $H_{L}^{p}(w)$ coincides with the classical weighted Hardy space $H^{p}(w)$. Therefore, in this particular case, we derive that the classical Riesz transform $\nabla(-\Delta)^{-1 / 2}$ is bounded on $H^{p}(w)$ for $\frac{n}{n+1}<p<1$, which was already obtained by Lee and Lin in [12].

\section{Notations and preliminaries}

First, let us recall some standard definitions and notations. The classical $A_{p}$ weight theory was first introduced by Muckenhoupt in the study of weighted $L^{p}$ boundedness of Hardy-Littlewood maximal functions in [13]. A weight $w$ is a locally integrable function on $\mathbb{R}^{n}$ which takes values in $(0, \infty)$ almost everywhere, $B=B\left(x_{0}, r\right)$ denotes the ball with the center $x_{0}$ and radius $r$. We say that $w \in A_{p}, 1<p<\infty$, if

$$
\left(\frac{1}{|B|} \int_{B} w(x) d x\right)\left(\frac{1}{|B|} \int_{B} w(x)^{-\frac{1}{p-1}} d x\right)^{p-1} \leq C \quad \text { for every ball } B \subseteq \mathbb{R}^{n},
$$

where $C$ is a positive constant which is independent of $B$.

For the case $p=1, w \in A_{1}$, if

$$
\frac{1}{|B|} \int_{B} w(x) d x \leq C \cdot \underset{x \in B}{\operatorname{essinf}} w(x) \quad \text { for every ball } B \subseteq \mathbb{R}^{n} .
$$

A weight function $w$ is said to belong to the reverse Hölder class $R H_{r}$ if there exist two constants $r>1$ and $C>0$ such that the following reverse Hölder inequality holds

$$
\left(\frac{1}{|B|} \int_{B} w(x)^{r} d x\right)^{1 / r} \leq C\left(\frac{1}{|B|} \int_{B} w(x) d x\right) \quad \text { for every ball } B \subseteq \mathbb{R}^{n} .
$$

If $w \in A_{p}$ with $1<p<\infty$, then we have $w \in A_{r}$ for all $r>p$, and $w \in A_{q}$ for some $1<q<p$. It follows from Hölder's inequality that $w \in R H_{r}$ implies $w \in R H_{s}$ for all $1<s<r$. Moreover, if $w \in R H_{r}, r>1$, then we have $w \in R H_{r+\varepsilon}$ for some $\varepsilon>0$.

Given a ball $B$ and $\lambda>0, \lambda B$ denotes the ball with the same center as $B$ whose radius is $\lambda$ times that of $B$. For a given weight function $w$, we 
denote the Lebesgue measure of $B$ by $|B|$ and the weighted measure of $B$ by $w(B)$, where $w(B)=\int_{B} w(x) d x$.

We give the following results that we will use in the sequel.

Lemma 2.1 ([8]). Let $w \in A_{p}, p \geq 1$. Then, for any ball $B$, there exists an absolute constant $C$ such that

$$
w(2 B) \leq C w(B) .
$$

In general, for any $\lambda>1$, we have

$$
w(\lambda B) \leq C \cdot \lambda^{n p} w(B),
$$

where $C$ does not depend on $B$ nor on $\lambda$.

Lemma $2.2([8,9])$. Let $w \in A_{p} \cap R H_{r}, p \geq 1$ and $r>1$. Then there exist constants $C_{1}, C_{2}>0$ such that

$$
C_{1}\left(\frac{|E|}{|B|}\right)^{p} \leq \frac{w(E)}{w(B)} \leq C_{2}\left(\frac{|E|}{|B|}\right)^{(r-1) / r}
$$

for any measurable subset $E$ of a ball $B$.

Given a Muckenhoupt's weight function $w$ on $\mathbb{R}^{n}$, for $0<p<\infty$, we denote by $L^{p}(w)$ the space of all functions satisfying

$$
\|f\|_{L^{p}(w)}=\left(\int_{\mathbb{R}^{n}}|f(x)|^{p} w(x) d x\right)^{1 / p}<\infty .
$$

Throughout this article, we will use $C$ to denote a positive constant, which is independent of the main parameters and not necessarily the same at each occurrence. By $A \sim B$, we mean that there exists a constant $C>1$ such that $\frac{1}{C} \leq \frac{A}{B} \leq C$. Moreover, we denote the conjugate exponent of $q>1$ by $q^{\prime}=q /(q-1)$.

\section{$3 \quad$ Weighted Hardy spaces $H_{L}^{p}(w)$ for $0<p<1$ and their atomic decompositions}

Let $L=-\Delta+V$. For any $t>0$, we define $P_{t}=e^{-t L}$ and

$$
Q_{t, k}=\left.(-t)^{k} \frac{d^{k} P_{s}}{d s^{k}}\right|_{s=t}=(t L)^{k} e^{-t L}, \quad k=1,2, \ldots
$$

We denote simply by $Q_{t}$ when $k=1$. First note that Gaussian upper bounds carry over from heat kernels to their time derivatives. 
Lemma $3.1([2,14])$. For every $k=1,2, \ldots$, there exist two positive constants $C_{k}$ and $c_{k}$ such that the kernel $p_{t, k}(x, y)$ of the operator $Q_{t, k}$ satisfies

$$
\left|p_{t, k}(x, y)\right| \leq \frac{C_{k}}{(4 \pi t)^{n / 2}} e^{-\frac{|x-y|^{2}}{c_{k} t}}
$$

for all $t>0$ and almost all $x, y \in \mathbb{R}^{n}$.

Set

$$
H^{2}\left(\mathbb{R}^{n}\right)=\overline{\mathcal{R}(L)}=\overline{\left\{L u \in L^{2}\left(\mathbb{R}^{n}\right): u \in L^{2}\left(\mathbb{R}^{n}\right)\right\}},
$$

where $\overline{\mathcal{R}(L)}$ stands for the range of $L$. We also set

$$
\Gamma(x)=\left\{(y, t) \in \mathbb{R}_{+}^{n+1}:|x-y|<t\right\} .
$$

For a given function $f \in L^{2}\left(\mathbb{R}^{n}\right)$, we consider the area integral function associated to Schrödinger operator $L$

$$
S_{L}(f)(x)=\left(\iint_{\Gamma(x)}\left|Q_{t^{2}}(f)(y)\right|^{2} \frac{d y d t}{t^{n+1}}\right)^{1 / 2}, \quad x \in \mathbb{R}^{n} .
$$

Given a weight function $w$ on $\mathbb{R}^{n}$, for $0<p<1$, we shall define the weighted Hardy spaces $H_{L}^{p}(w)$ as the completion of $H^{2}\left(\mathbb{R}^{n}\right)$ in the norm given by the $L^{p}(w)$-norm of area integral function; that is

$$
\|f\|_{H_{L}^{p}(w)}=\left\|S_{L}(f)\right\|_{L^{p}(w)} .
$$

Let $M \in \mathbb{N}$ and $0<p<1$. As in [18], we say that a function $a(x) \in L^{2}\left(\mathbb{R}^{n}\right)$ is called a $(p, M)$-atom with respect to $w$ (or a $w$-( $p, M)$-atom) if there exist a ball $B=B\left(x_{0}, r\right)$ and a function $b \in \mathcal{D}\left(L^{M}\right)$ such that

(a) $a=L^{M} b$;

(b) $\operatorname{supp} L^{k} b \subseteq B, \quad k=0,1, \ldots, M$;

(c) $\left\|\left(r^{2} L\right)^{k} b\right\|_{L^{2}(B)} \leq r^{2 M}|B|^{1 / 2} w(B)^{-1 / p}, \quad k=0,1, \ldots, M$.

Let $M \in \mathbb{N}$ and $\frac{n}{n+1}<p<1$. For any $w$ - $(p, M)$-atom $a$ associated to a ball $B=B\left(x_{0}, r\right),\|a\|_{L^{2}(B)} \leq|B|^{1 / 2} w(B)^{-1 / p}$, we will show that $a \in H_{L}^{p}(w)$ and its $H_{L}^{p}(w)$-norm is uniformly bounded; precisely

Theorem 3.2. Let $M \in \mathbb{N}, \frac{n}{n+1}<p<1$ and $w \in A_{1} \cap R H_{(2 / p)^{\prime}}$. Then there exists a constant $C>0$ independent of a such that

$$
\left\|S_{L}(a)\right\|_{L^{p}(w)} \leq C .
$$


Proof. We write

$$
\begin{aligned}
\left\|S_{L}(a)\right\|_{L^{p}(w)}^{p} & =\int_{2 B}\left|S_{L}(a)(x)\right|^{p} w(x) d x+\int_{(2 B)^{c}}\left|S_{L}(a)(x)\right|^{p} w(x) d x \\
& =I_{1}+I_{2} .
\end{aligned}
$$

Set $q=2 / p$. Note that $w \in R H_{q^{\prime}}$, then it follows from Hölder's inequality, Lemma 2.1 and the $L^{2}$ boundedness of $S_{L}$ (see (3.2) below) that

$$
\begin{aligned}
I_{1} & \leq\left(\int_{2 B}\left|S_{L}(a)(x)\right|^{2} d x\right)^{p / 2}\left(\int_{2 B} w(x)^{q^{\prime}} d x\right)^{1 / q^{\prime}} \\
& \leq C\|a\|_{L^{2}(B)}^{p} \cdot \frac{w(2 B)}{|2 B|^{1 / q}} \\
& \leq C .
\end{aligned}
$$

We turn to deal with $I_{2}$. By using Hölder's inequality and the fact that $w \in R H_{q^{\prime}}$, we can get

$$
\begin{aligned}
I_{2} & =\sum_{k=1}^{\infty} \int_{2^{k+1} B \backslash 2^{k} B}\left|S_{L}(a)(x)\right|^{p} w(x) d x \\
& \leq C \sum_{k=1}^{\infty}\left(\int_{2^{k+1} B \backslash 2^{k} B}\left|S_{L}(a)(x)\right|^{2} d x\right)^{p / 2} \cdot \frac{w\left(2^{k+1} B\right)}{\left|2^{k+1} B\right|^{1 / q}} .
\end{aligned}
$$

For any $x \in 2^{k+1} B \backslash 2^{k} B, k=1,2, \ldots$, we write

$$
\begin{aligned}
& \left|S_{L}(a)(x)\right|^{2} \\
= & \int_{0}^{r} \int_{|y-x|<t}\left|t^{2} L e^{-t^{2} L} a(y)\right|^{2} \frac{d y d t}{t^{n+1}}+\int_{r}^{\infty} \int_{|y-x|<t}\left|t^{2} L e^{-t^{2} L} a(y)\right|^{2} \frac{d y d t}{t^{n+1}} \\
= & \mathrm{I}+\mathrm{II} .
\end{aligned}
$$

For the term I, note that $0<t<r$. By a simple calculation, we obtain that for any $(y, t) \in \Gamma(x), x \in 2^{k+1} B \backslash 2^{k} B, z \in B$, then $|y-z| \geq 2^{k-1} r$. Hence, by using Hölder's inequality and Lemma 3.1, we deduce

$$
\begin{aligned}
\left|t^{2} L e^{-t^{2} L} a(y)\right| & \leq C \cdot \frac{t}{\left(2^{k-1} r\right)^{n+1}} \int_{B}|a(z)| d z \\
& \leq C \cdot \frac{t}{\left(2^{k} r\right)^{n+1}}\|a\|_{L^{2}\left(\mathbb{R}^{n}\right)}|B|^{1 / 2} \\
& \leq C \cdot w(B)^{-1 / p} \frac{t}{2^{k(n+1)} \cdot r} .
\end{aligned}
$$


Consequently

$$
\begin{aligned}
\mathrm{I} & \leq C\left(\frac{1}{2^{k(n+1)} w(B)^{1 / p}}\right)^{2} \cdot \frac{1}{r^{2}} \int_{0}^{r} t d t \\
& \leq C\left(\frac{1}{2^{k(n+1)} w(B)^{1 / p}}\right)^{2} .
\end{aligned}
$$

We now estimate the other term II. In this case, a direct computation shows that for any $(y, t) \in \Gamma(x), x \in 2^{k+1} B \backslash 2^{k} B$ and $z \in B$, we have $t+|y-z| \geq$ $2^{k-1} r$. Since there exists a function $b \in \mathcal{D}\left(L^{M}\right)$ such that $a=L^{M} b$, then by Hölder's inequality and Lemma 3.1 again, we get

$$
\begin{aligned}
\left|t^{2} L e^{-t^{2} L} a(y)\right| & =\left|\left(t^{2} L\right)^{M+1} e^{-t^{2} L} b(y)\right| \cdot \frac{1}{t^{2 M}} \\
& \leq C \cdot \frac{1}{\left(2^{k-1} r\right)^{n+1}} \int_{B}|b(z)| d z \cdot \frac{1}{t^{2 M-1}} \\
& \leq C \cdot \frac{1}{\left(2^{k} r\right)^{n+1}}\|b\|_{L^{2}\left(\mathbb{R}^{n}\right)}|B|^{1 / 2} \cdot \frac{1}{t^{2 M-1}} \\
& \leq C \cdot \frac{r^{2 M-1}}{2^{k(n+1)} w(B)^{1 / p}} \cdot \frac{1}{t^{2 M-1}} .
\end{aligned}
$$

Therefore

$$
\begin{aligned}
\mathrm{II} & \leq C\left(\frac{1}{2^{k(n+1)} w(B)^{1 / p}}\right)^{2} \cdot r^{4 M-2} \int_{r}^{\infty} \frac{d t}{t^{4 M-1}} \\
& \leq C\left(\frac{1}{2^{k(n+1)} w(B)^{1 / p}}\right)^{2} .
\end{aligned}
$$

Combining the above estimates for I and II, we thus obtain

$$
\left|S_{L}(a)(x)\right| \leq C \cdot \frac{1}{2^{k(n+1)} w(B)^{1 / p}}, \quad \text { when } x \in 2^{k+1} B \backslash 2^{k} B .
$$

Then it follows immediately from Lemma 2.1 that

$$
\begin{aligned}
I_{2} & \leq C \sum_{k=1}^{\infty} \frac{1}{2^{k p(n+1)} w(B)} \cdot w\left(2^{k+1} B\right) \\
& \leq C \sum_{k=1}^{\infty} \frac{1}{2^{k p(n+1)-k n}} \\
& \leq C
\end{aligned}
$$

where in the last inequality we have used the fact that $p>n /(n+1)$. Summarizing the estimates for $I_{1}$ and $I_{2}$ derived above, we complete the proof of Theorem 3.2. 
For every bounded Borel function $F:[0, \infty) \rightarrow \mathbb{C}$, we define the operator $F(L): L^{2}\left(\mathbb{R}^{n}\right) \rightarrow L^{2}\left(\mathbb{R}^{n}\right)$ by the following formula

$$
F(L)=\int_{0}^{\infty} F(\lambda) d E_{L}(\lambda)
$$

where $E_{L}(\lambda)$ is the spectral decomposition of $L$. Therefore, the operator $\cos (t \sqrt{L})$ is well-defined on $L^{2}\left(\mathbb{R}^{n}\right)$. Moreover, it follows from [16] that there exists a constant $c_{0}$ such that the Schwartz kernel $K_{\cos (t \sqrt{L})}(x, y)$ of $\cos (t \sqrt{L})$ has support contained in $\left\{(x, y) \in \mathbb{R}^{n} \times \mathbb{R}^{n}:|x-y| \leq c_{0} t\right\}$. By the functional calculus for $L$ and Fourier inversion formula, whenever $F$ is an even bounded Borel function with $\hat{F} \in L^{1}(\mathbb{R})$, we can write

$$
F(\sqrt{L})=(2 \pi)^{-1} \int_{-\infty}^{\infty} \hat{F}(t) \cos (t \sqrt{L}) d t
$$

Lemma $3.3([10])$. Let $\varphi \in C_{0}^{\infty}(\mathbb{R})$ be even and supp $\varphi \subseteq\left[-c_{0}^{-1}, c_{0}^{-1}\right]$. Let $\Phi$ denote the Fourier transform of $\varphi$. Then for each $j=0,1, \ldots$, and for all $t>0$, the Schwartz kernel $K_{\left(t^{2} L\right)^{j} \Phi(t \sqrt{L})}(x, y)$ of $\left(t^{2} L\right)^{j} \Phi(t \sqrt{L})$ satisfies

$$
\operatorname{supp} K_{\left(t^{2} L\right)^{j} \Phi(t \sqrt{L})} \subseteq\left\{(x, y) \in \mathbb{R}^{n} \times \mathbb{R}^{n}:|x-y| \leq t\right\} .
$$

For a given $s>0$, we set

$$
\mathcal{F}(s)=\left\{\psi: \mathbb{C} \rightarrow \mathbb{C} \text { measurable, }|\psi(z)| \leq C \frac{|z|^{s}}{1+|z|^{2 s}}\right\}
$$

Then for any nonzero function $\psi \in \mathcal{F}(s)$, we have the following estimate(see [18])

$$
\left(\int_{0}^{\infty}\|\psi(t \sqrt{L}) f\|_{L^{2}\left(\mathbb{R}^{n}\right)}^{2} \frac{d t}{t}\right)^{1 / 2} \leq C\|f\|_{L^{2}\left(\mathbb{R}^{n}\right)} .
$$

In particular, we have

$$
\left\|S_{L}(f)\right\|_{L^{2}\left(\mathbb{R}^{n}\right)} \leq C\|f\|_{L^{2}\left(\mathbb{R}^{n}\right)} .
$$

We are going to establish the atomic decomposition for the weighted Hardy spaces $H_{L}^{p}(w)(0<p<1)$.

Theorem 3.4. Let $M \in \mathbb{N}, 0<p<1$ and $w \in A_{1}$. If $f \in H_{L}^{p}(w)$, then there exist a family of $w$ - $(p, M)$-atoms $\left\{a_{j}\right\}$ and a sequence of real numbers $\left\{\lambda_{j}\right\}$ with $\sum_{j}\left|\lambda_{j}\right|^{p} \leq C\|f\|_{H_{L}^{p}(w)}^{p}$ such that $f$ can be represented in the form $f(x)=\sum_{j} \lambda_{j} a_{j}(x)$, and the sum converges both in the sense of $L^{2}\left(\mathbb{R}^{n}\right)$-norm and $H_{L}^{p}(w)$-norm. 
Proof. First assume that $f \in H_{L}^{p}(w) \cap H^{2}\left(\mathbb{R}^{n}\right)$. We follow the same constructions as in [18]. Let $\varphi$ and $\Phi$ be as in Lemma 3.3. We set $\Psi(x)=$ $x^{2 M} \Phi(x), x \in \mathbb{R}$. By the $L^{2}$-functional calculus of $L$, for every $f \in H^{2}\left(\mathbb{R}^{n}\right)$, we can establish the following version of the Calderón reproducing formula

$$
f(x)=c_{\psi} \int_{0}^{\infty} \Psi(t \sqrt{L}) t^{2} L e^{-t^{2} L}(f)(x) \frac{d t}{t},
$$

where the above equality holds in the sense of $L^{2}\left(\mathbb{R}^{n}\right)$-norm. For any $k \in \mathbb{Z}$, set

$$
\Omega_{k}=\left\{x \in \mathbb{R}^{n}: S_{L, 10 \sqrt{n}}(f)(x)>2^{k}\right\} .
$$

Let $\mathbb{D}$ denote the set formed by all dyadic cubes in $\mathbb{R}^{n}$ and let

$$
\mathbb{D}_{k}=\left\{Q \in \mathbb{D}: w\left(Q \cap \Omega_{k}\right)>\frac{w(Q)}{2}, w\left(Q \cap \Omega_{k+1}\right) \leq \frac{w(Q)}{2}\right\} .
$$

Obviously, for any $Q \in \mathbb{D}$, there exists a unique $k \in \mathbb{Z}$ such that $Q \in \mathbb{D}_{k}$. We also denote the maximal dyadic cubes in $\mathbb{D}_{k}$ by $Q_{k}^{l}$. Note that the maximal dyadic cubes $Q_{k}^{l}$ are pairwise disjoint, then it is easy to check that

$$
\sum_{l} w\left(Q_{k}^{l}\right) \leq C \cdot w\left(\Omega_{k}\right)
$$

Set

$$
\widetilde{Q}=\left\{(y, t) \in \mathbb{R}_{+}^{n+1}: y \in Q, \frac{l(Q)}{2}<t \leq l(Q)\right\}
$$

where $l(Q)$ denotes the side length of $Q$. If we set $\widetilde{Q_{k}^{l}}=\bigcup_{Q_{k}^{l} \supseteq Q \in \mathbb{D}_{k}} \widetilde{Q}$, then we have $\mathbb{R}_{+}^{n+1}=\bigcup_{k} \bigcup_{l} \widetilde{Q_{k}^{l}}$. Hence, by the formula (3.3), we can write

$$
f(x)=\sum_{k} \sum_{l} c_{\psi} \int_{\widetilde{Q_{k}^{l}}} \Psi(t \sqrt{L})(x, y) t^{2} L e^{-t^{2} L} f(y) \frac{d y d t}{t}=\sum_{k} \sum_{l} \lambda_{k l} a_{k}^{l}(x),
$$

where $a_{k}^{l}=L^{M} b_{k}^{l}$,

$$
b_{k}^{l}(x)=c_{\psi} \lambda_{k l}^{-1} \int_{{\widetilde{Q_{k}^{l}}}^{l}} t^{2 M} \Phi(t \sqrt{L})(x, y) t^{2} L e^{-t^{2} L} f(y) \frac{d y d t}{t}
$$

and

$$
\lambda_{k l}=w\left(Q_{k}^{l}\right)^{1 / p-1 / 2}\left(\int_{\widetilde{Q_{k}^{l}}}\left|t^{2} L e^{-t^{2} L} f(y)\right|^{2} \frac{w\left(Q_{k}^{l}\right)}{\left|Q_{k}^{l}\right|} \frac{d y d t}{t}\right)^{1 / 2} .
$$


By using Lemma 3.3, the authors in [18] showed that for every $j=0,1, \ldots, M$, $\operatorname{supp}\left(L^{j} b_{k}^{l}\right) \subseteq 3 Q_{k}^{l}$. In [18], they also obtained the following estimate

$$
\sum_{l} \int_{\widetilde{Q_{k}^{l}}}\left|t^{2} L e^{-t^{2} L} f(y)\right|^{2} \frac{w\left(Q_{k}^{l}\right)}{\left|Q_{k}^{l}\right|} \frac{d y d t}{t} \leq C \cdot 2^{2 k} w\left(\Omega_{k}\right) .
$$

Since

$$
\left\|\left(l\left(Q_{k}^{l}\right)^{2} L\right)^{j} b_{k}^{l}\right\|_{L^{2}\left(3 Q_{k}^{l}\right)}=\sup _{\|h\|_{L^{2}\left(3 Q_{k}^{l}\right)} \leq 1}\left|\int_{\mathbb{R}^{n}}\left(l\left(Q_{k}^{l}\right)^{2} L\right)^{j} b_{k}^{l}(x) h(x) d x\right| .
$$

Let $\Psi_{j}(x)=x^{2 j} \Phi(x), j=0,1, \ldots, M$. Then we can easily verify that $\Psi_{j} \in \mathcal{F}(2 j)$. Observe that when $(y, t) \in \widetilde{Q_{k}^{l}}$, we have $t \sim l\left(Q_{k}^{l}\right)$. Then it follows from Hölder's inequality and the estimate (3.1) that

$$
\begin{aligned}
& \left|\int_{\mathbb{R}^{n}}\left(l\left(Q_{k}^{l}\right)^{2} L\right)^{j} b_{k}^{l}(x) h(x) d x\right| \\
\leq & \frac{C \cdot l\left(Q_{k}^{l}\right)^{2 M}}{\lambda_{k l}}\left(\int_{\widetilde{Q_{k}^{l}}}\left|t^{2} L e^{-t^{2} L} f(y)\right|^{2} \frac{d y d t}{t}\right)^{1 / 2} \\
& \times\left(\int_{\widetilde{Q_{k}^{l}}}\left|\left(t^{2} L\right)^{j} \Phi(t \sqrt{L})\left(h \chi_{3 Q_{k}^{l}}\right)(y)\right|^{2} \frac{d y d t}{t}\right)^{1 / 2} \\
\leq & C \cdot l\left(Q_{k}^{l}\right)^{2 M}\left|Q_{k}^{l}\right|^{1 / 2} w\left(Q_{k}^{l}\right)^{-1 / p}\left(\int_{0}^{\infty}\left\|\Psi_{j}(t \sqrt{L})\left(h \chi_{3 Q_{k}^{l}}\right)\right\|_{L^{2}\left(\mathbb{R}^{n}\right)}^{2} \frac{d t}{t}\right)^{1 / 2} \\
\leq & C \cdot l\left(Q_{k}^{l}\right)^{2 M}\left|Q_{k}^{l}\right|^{1 / 2} w\left(Q_{k}^{l}\right)^{-1 / p} .
\end{aligned}
$$

Hence

$$
\left\|\left(l\left(Q_{k}^{l}\right)^{2} L\right)^{j} b_{k}^{l}\right\|_{L^{2}\left(3 Q_{k}^{l}\right)} \leq C \cdot l\left(Q_{k}^{l}\right)^{2 M}\left|Q_{k}^{l}\right|^{1 / 2} w\left(Q_{k}^{l}\right)^{-1 / p} .
$$

From the above discussions, we have proved that these functions $a_{k}^{l}$ are all $w$ - $(p, M)$-atoms up to a normalization by a multiplicative constant. Finally, by using Hölder's inequality, the estimates (3.4) and (3.5), we obtain

$$
\begin{aligned}
\sum_{k} \sum_{l}\left|\lambda_{k l}\right|^{p} & =\sum_{k} \sum_{l}\left(w\left(Q_{k}^{l}\right)\right)^{1-p / 2}\left(\int_{\widetilde{Q_{k}^{l}}}\left|t^{2} L e^{-t^{2} L} f(y)\right|^{2} \frac{w\left(Q_{k}^{l}\right)}{\left|Q_{k}^{l}\right|} \frac{d y d t}{t}\right)^{p / 2} \\
& \leq \sum_{k}\left(\sum_{l} w\left(Q_{k}^{l}\right)\right)^{1-p / 2}\left(\sum_{l} \int_{\widetilde{Q_{k}^{l}}}\left|t^{2} L e^{-t^{2} L} f(y)\right|^{2} \frac{w\left(Q_{k}^{l}\right)}{\left|Q_{k}^{l}\right|} \frac{d y d t}{t}\right)^{p / 2} \\
& \leq C \sum_{k}\left(w\left(\Omega_{k}\right)\right)^{1-p / 2}\left(2^{2 k} w\left(\Omega_{k}\right)\right)^{p / 2} \\
& \leq C\left\|S_{L}(f)\right\|_{L^{p}(w)}^{p} .
\end{aligned}
$$


Therefore, we have established the atomic decomposition for all functions in the space $H_{L}^{p}(w) \cap H^{2}\left(\mathbb{R}^{n}\right)$. By a standard density argument, we can show that the same conclusion holds for $H_{L}^{p}(w)$. Following along the same arguments as in [18], we can also prove that the sum $f=\sum_{j} \lambda_{j} a_{j}$ converges both in the sense of $L^{2}\left(\mathbb{R}^{n}\right)$-norm and $H_{L}^{p}(w)$-norm, the details are omitted here. This completes the proof of Theorem 3.4.

\section{Proof of Theorem 1.1}

We shall need the following Davies-Gaffney estimate which can be found in $[10,18]$.

Lemma 4.1. For any two closed sets $E$ and $F$ of $\mathbb{R}^{n}$, there exist two positive constants $C$ and $c$ such that

$$
\left\|t \nabla e^{-t^{2} L} f\right\|_{L^{2}(F)} \leq C \cdot e^{-\frac{d i s t(E, F)^{2}}{c t^{2}}}\|f\|_{L^{2}(E)}
$$

for every $f \in L^{2}\left(\mathbb{R}^{n}\right)$ with support contained in $E$.

Theorem 4.2. Let $\frac{n}{n+1}<p<1$ and $w \in A_{1} \cap R H_{(2 / p)^{\prime}}$. Then the operator $\nabla L^{-1 / 2}$ is bounded from $H_{L}^{p}(w)$ to $L^{p}(w)$.

Proof. By Theorem 3.4 we just proved, it is enough for us to show that for any $w$ - $(p, M)$-atom $a, M>\frac{n}{2}\left(\frac{1}{p}-\frac{1}{2}\right)$, there exists a constant $C>0$ independent of $a$ such that $\left\|\nabla L^{-1 / 2}(a)\right\|_{L^{p}(w)} \leq C$. Let $a$ be a $w$ - $(p, M)$ atom with supp $a \subseteq B=B\left(x_{0}, r\right),\|a\|_{L^{2}(B)} \leq|B|^{1 / 2} w(B)^{-1 / p}$. We write

$$
\begin{aligned}
\left\|\nabla L^{-1 / 2}(a)\right\|_{L^{p}(w)}^{p} & =\int_{2 B}\left|\nabla L^{-1 / 2}(a)(x)\right|^{p} w(x) d x+\int_{(2 B)^{c}}\left|\nabla L^{-1 / 2}(a)(x)\right|^{p} w(x) d x \\
& =J_{1}+J_{2} .
\end{aligned}
$$

Set $q=2 / p$. Applying Hölder's inequality, the $L^{2}$ boundedness of $\nabla L^{-1 / 2}$, Lemma 2.1 and $w \in R H_{q^{\prime}}$, we thus have

$$
\begin{aligned}
J_{1} & \leq\left(\int_{2 B}\left|\nabla L^{-1 / 2}(a)(x)\right|^{2} d x\right)^{p / 2}\left(\int_{2 B} w(x)^{q^{\prime}} d x\right)^{1 / q^{\prime}} \\
& \leq C\|a\|_{L^{2}(B)}^{p} \cdot \frac{w(2 B)}{|2 B|^{1 / q}} \\
& \leq C .
\end{aligned}
$$


On the other hand, it follows from Hölder's inequality and $w \in R H_{q^{\prime}}$ that

$$
\begin{aligned}
J_{2} & =\sum_{k=1}^{\infty} \int_{2^{k+1} B \backslash 2^{k} B}\left|\nabla L^{-1 / 2}(a)(x)\right|^{p} w(x) d x \\
& \leq C \sum_{k=1}^{\infty}\left(\int_{2^{k+1} B \backslash 2^{k} B}\left|\nabla L^{-1 / 2}(a)(x)\right|^{2} d x\right)^{p / 2} \cdot \frac{w\left(2^{k+1} B\right)}{\left|2^{k+1} B\right|^{1 / q}} .
\end{aligned}
$$

By a change of variable $s=t^{2}$, we can rewrite (1.2) as

$$
\nabla L^{-1 / 2}(a)(x)=\frac{2}{\sqrt{\pi}} \int_{0}^{\infty} s \nabla e^{-s^{2} L}(a)(x) \frac{d s}{s} .
$$

For any $k=1,2, \ldots$, it follows immediately from Minkowski's integral inequality that

$$
\begin{aligned}
& \left(\int_{2^{k+1} B \backslash 2^{k} B}\left|\nabla L^{-1 / 2}(a)(x)\right|^{2} d x\right)^{1 / 2} \\
\leq & C \int_{0}^{r}\left\|s \nabla e^{-s^{2} L} a\right\|_{L^{2}\left(2^{k+1} B \backslash 2^{k} B\right)} \frac{d s}{s}+C \int_{r}^{\infty}\left\|s \nabla e^{-s^{2} L} a\right\|_{L^{2}\left(2^{k+1} B \backslash 2^{k} B\right)} \frac{d s}{s} \\
= & \mathrm{III}+\mathrm{IV} .
\end{aligned}
$$

Observe that $M>\frac{n}{2}\left(\frac{1}{p}-\frac{1}{2}\right)$. Then we are able to choose a positive number $N$ such that $\frac{n}{2}\left(\frac{1}{p}-\frac{1}{2}\right)<N<M$. By using Lemma 4.1, we can get

$$
\begin{aligned}
\mathrm{III} & \leq C \int_{0}^{r} e^{-\frac{\left(2^{k} r\right)^{2}}{s^{2}}}\|a\|_{L^{2}(B)} \frac{d s}{s} \\
& \leq C \int_{0}^{r} \frac{s^{2 N}}{\left(2^{k} r\right)^{2 N}} \frac{d s}{s} \cdot\|a\|_{L^{2}(B)} \\
& \leq C \cdot 2^{-2 k N}|B|^{1 / 2} w(B)^{-1 / p} .
\end{aligned}
$$

We now turn to estimate the term IV. Since $a=L^{M} b$ and $\|b\|_{L^{2}(B)} \leq$ $r^{2 M}|B|^{1 / 2} w(B)^{-1 / p}$. Using Lemma 3.1 and Lemma 4.1, we deduce

$$
\begin{aligned}
\mathrm{IV} & =C \int_{r}^{\infty}\left\|s \nabla e^{-s^{2} L}\left(L^{M} b\right)\right\|_{L^{2}\left(2^{k+1} B \backslash 2^{k} B\right)} \frac{d s}{s} \\
& =C \int_{r}^{\infty}\left\|s \nabla e^{-\frac{s^{2} L}{2}}\left(s^{2} L\right)^{M} e^{-\frac{s^{2} L}{2}} b\right\|_{L^{2}\left(2^{k+1} B \backslash 2^{k} B\right)} \frac{d s}{s^{2 M+1}} \\
& \leq C \int_{r}^{\infty} e^{-\frac{\left(2^{k} r\right)^{2}}{s^{2}}}\left\|\left(s^{2} L\right)^{M} e^{-\frac{s^{2} L}{2}} b\right\|_{L^{2}(B)} \frac{d s}{s^{2 M+1}} \\
& \leq C \int_{r}^{\infty} \frac{s^{2 N}}{\left(2^{k} r\right)^{2 N}} \frac{d s}{s^{2 M+1}} \cdot\|b\|_{L^{2}(B)} \\
& \leq C \cdot 2^{-2 k N}|B|^{1 / 2} w(B)^{-1 / p} .
\end{aligned}
$$


Combining the above inequality (4.4) with (4.3), we thus obtain

$$
\left(\int_{2^{k+1} B \backslash 2^{k} B}\left|\nabla L^{-1 / 2}(a)(x)\right|^{2} d x\right)^{1 / 2} \leq C \cdot 2^{-2 k N}|B|^{1 / 2} w(B)^{-1 / p} .
$$

Substituting the above inequality (4.5) into (4,1) and using Lemma 2.1, then we have

$$
\begin{aligned}
J_{2} & \leq C \sum_{k=1}^{\infty}\left(2^{-2 k N}|B|^{1 / 2} w(B)^{-1 / p}\right)^{p} \cdot \frac{w\left(2^{k+1} B\right)}{\left|2^{k+1} B\right|^{p / 2}} \\
& \leq C \sum_{k=1}^{\infty} \frac{1}{2^{k\left(2 p N+\frac{n p}{2}-n\right)}} \\
& \leq C
\end{aligned}
$$

where the last series is convergent since $N>\frac{n}{2}\left(\frac{1}{p}-\frac{1}{2}\right)$. Summarizing the estimates for $J_{1}$ and $J_{2}$, we get the desired result.

The real-variable theory of classical weighted Hardy spaces have been extensively studied by many authors. In 1979, Garcia-Cuerva studied the atomic decomposition and the dual spaces of $H^{p}(w)$ for $0<p \leq 1$. In 2002, Lee and Lin gave the molecular characterization of $H^{p}(w)$ for $0<p \leq 1$, they also obtained the $H^{p}(w)\left(\frac{1}{2}<p \leq 1\right)$ boundedness of the Hilbert transform and the $H^{p}(w)\left(\frac{n}{n+1}<p \leq 1\right)$ boundedness of the Riesz transforms. For the results mentioned above, we refer the readers to $[7,12,19]$ for further details.

Let $\frac{n}{n+1}<p<1$ and $w \in A_{1}$. A real-valued function $a(x)$ is called a $w$-(p,2,0)-atom if the following conditions are satisfied(see $[7,19])$ :

(a) supp $a \subseteq B$;

(b) $\|a\|_{L^{2}(B)} \leq|B|^{1 / 2} w(B)^{-1 / p}$;

(c) $\int_{\mathbb{R}^{n}} a(x) d x=0$.

Theorem 4.3. Let $\frac{n}{n+1}<p<1$ and $w \in A_{1}$. For each $f \in H^{p}(w)$, there exist a family of $w$ - $(p, 2,0)$-atoms $\left\{a_{j}\right\}$ and a sequence of real numbers $\left\{\lambda_{j}\right\}$ with $\sum_{j}\left|\lambda_{j}\right|^{p} \leq C\|f\|_{H^{p}(w)}^{p}$ such that $f=\sum_{j} \lambda_{j} a_{j}$ in the sense of $H^{p}(w)$ norm.

Next, as in [18], we shall also define the new weighted molecules for $H^{p}(w)$. Let $\frac{n}{n+1}<p<1, w \in A_{1}$ and $\varepsilon>0$. A function $m(x) \in L^{2}\left(\mathbb{R}^{n}\right)$ is called a $w$ - $(p, 2,0, \varepsilon)$-molecule associated to a ball $B$ if the following conditions are satisfied:

(A) $\int_{\mathbb{R}^{n}} m(x) d x=0$;

(B) $\|m\|_{L^{2}(2 B)} \leq|B|^{1 / 2} w(B)^{-1 / p}$; 
(C) $\|m\|_{L^{2}\left(2^{k+1} B \backslash 2^{k} B\right)} \leq 2^{-k \varepsilon}\left|2^{k} B\right|^{1 / 2} w\left(2^{k} B\right)^{-1 / p}, \quad k=1,2, \ldots$

Note that for every $w$ - $(p, 2,0)$-atom, it is a $w$ - $(p, 2,0, \varepsilon)$-molecule for all $\varepsilon>0$. Then we are able to establish the following molecular characterization for the classical weighted Hardy spaces $H^{p}(w)$.

Theorem 4.4. Let $\frac{n}{n+1}<p<1$ and $w \in A_{1}$.

(i) If $f \in H^{p}(w)$, then there exist a family of $w-(p, 2,0, \varepsilon)$-molecules $\left\{m_{j}\right\}$ and a sequence of real numbers $\left\{\lambda_{j}\right\}$ with $\sum_{j}\left|\lambda_{j}\right|^{p} \leq C\|f\|_{H^{p}(w)}^{p}$ such that $f=\sum_{j} \lambda_{j} a_{j}$ in the sense of $H^{p}(w)$ norm.

(ii) Suppose that $w \in R H_{(2 / p)^{\prime}}$ and $\varepsilon>n / 2$, then every $w$ - $(p, 2,0, \varepsilon)$ molecule $m$ is in $H^{p}(w)$. Moreover, there exists a constant $C>0$ independent of $m$ such that $\|m\|_{H^{p}(w)} \leq C$.

Proof. $(i)$ is a straightforward consequence of Theorem 4.3.

(ii) We follow the idea of [18]. Denote $m_{0}(x)=m(x) \chi_{2 B}(x), m_{k}(x)=$ $m(x) \chi_{2^{k+1}{ }_{B \backslash 2^{k} B}}(x), k=1,2, \ldots$ Then we can decompose $m(x)$ as

$$
m(x)=\sum_{k=0}^{\infty} m_{k}(x)=\sum_{k=0}^{\infty}\left(m_{k}(x)-N_{k}(x)\right)+\sum_{k=0}^{\infty} N_{k}(x),
$$

where $N_{0}(x)=\frac{1}{|2 B|} \int_{\mathbb{R}^{n}} m_{0}(y) d y \cdot \chi_{2 B}(x)$ and $N_{k}(x)=\frac{1}{\left|2^{k+1} B \backslash 2^{k} B\right|} \int_{\mathbb{R}^{n}} m_{k}(y) d y$. $\chi_{2^{k+1}{ }_{B \backslash 2^{k} B}}(x), k=1,2, \ldots$ Following along the same lines as in [18], we can also show that each $\left(m_{k}-N_{k}\right)$ is a multiple of $w$ - $(p, 2,0)$-atom with a sequence of coefficients in $l^{p}$. We set $\eta_{k}=\int_{\mathbb{R}^{n}} m_{k}(y) d y, k=0,1, \ldots$ In [18], Song and Yan established the following identity

$$
\sum_{k=0}^{\infty} N_{k}(x)=\sum_{k=0}^{\infty} p_{k} \cdot \psi_{k}(x)
$$

where $p_{k}=\sum_{j=k+1}^{\infty} \eta_{j}$ and $\psi_{k}(x)=\frac{N_{k+1}(x)}{\eta_{k+1}}-\frac{N_{k}(x)}{\eta_{k}}$. Then we have

$$
\begin{aligned}
\left|p_{k}\right| & \leq \sum_{j=k+1}^{\infty} \int_{2^{j+1} B \backslash 2^{j} B}|m(y)| d y \\
& \leq \sum_{j=k+1}^{\infty}\|m\|_{L^{2}\left(2^{j+1} B \backslash 2^{j} B\right)}\left|2^{j+1} B\right|^{1 / 2} \\
& \leq C \sum_{j=k+1}^{\infty} 2^{-j \varepsilon} \cdot\left|2^{j} B\right| w\left(2^{j} B\right)^{-1 / p} .
\end{aligned}
$$


When $j \geq k+1$, then $2^{k} B \subseteq 2^{j} B$. Since $w \in R H_{(2 / p)^{\prime}}$, then by Lemma 2.2, we can get

$$
\frac{w\left(2^{k} B\right)}{w\left(2^{j} B\right)} \leq C\left(\frac{\left|2^{k} B\right|}{\left|2^{j} B\right|}\right)^{p / 2}
$$

Hence

$$
\begin{aligned}
\left|p_{k}\right| & \leq C \cdot \frac{\left|2^{k} B\right|}{w\left(2^{k} B\right)^{1 / p}} \sum_{j=k+1}^{\infty} 2^{-j \varepsilon}\left(\frac{\left|2^{j} B\right|}{\left|2^{k} B\right|}\right)^{1 / 2} \\
& \leq C \cdot \frac{\left|2^{k} B\right|}{w\left(2^{k} B\right)^{1 / p}}\left(\sum_{j=k+1}^{\infty} 2^{-j(\varepsilon-n / 2)}\right) \cdot 2^{-k n / 2} \\
& \leq C \cdot 2^{-k \varepsilon} \frac{\left|2^{k} B\right|}{w\left(2^{k} B\right)^{1 / p}}
\end{aligned}
$$

where the last inequality holds since $\varepsilon>n / 2$. As in [18], we can easily check that $2^{k \varepsilon} p_{k} \psi(x)$ are all $w$ - $(p, 2,0)$-atoms associated to $2^{k+1} B$. Therefore the sum $\sum_{k=0}^{\infty} N_{k}$ can be write as an infinite linear combination of $w$ $(p, 2,0)$-atoms with a sequence of coefficients in $l^{p}$. Summarizing the above discussions, we complete the proof of Theorem 4.4.

We are now in a position to give the proof of Theorem 1.1.

Proof of Theorem 1.1. By Theorem 3.4 and Theorem 4.4, it suffices to show that for every $w$ - $(p, M)$-atom $a$ with supp $a \subseteq B$, then $\nabla L^{-1 / 2} a$ is a $w$ $(p, 2,0, \varepsilon)$-molecule, where $M>n\left(\frac{1}{p}-\frac{1}{2}\right)$ and $\varepsilon>n / 2$. It is easy to see that $\int_{\mathbb{R}^{n}} \nabla L^{-1 / 2} a(x) d x=0$. It remains to verify the estimates (B) and (C). Hölder's inequality and the definition of $w$ - $(p, M)$-atom imply

$$
\left\|\nabla L^{-1 / 2}(a)\right\|_{L^{2}(2 B)} \leq C\|a\|_{L^{2}(B)} \leq C \cdot|B|^{1 / 2} w(B)^{-1 / p} .
$$

For $k=1,2, \ldots$, it follows from the previous estimates (4.3) and (4.4) that

$$
\left\|\nabla L^{-1 / 2}(a)\right\|_{L^{2}\left(2^{k+1} B \backslash 2^{k} B\right)} \leq C \cdot 2^{-2 k N}|B|^{1 / 2} w(B)^{-1 / p},
$$

where $N>0$ is chosen such that $n\left(\frac{1}{p}-\frac{1}{2}\right)<N<M$. By using Lemma 2.2, we get

Hence

$$
\frac{w(B)}{w\left(2^{k} B\right)} \geq C \cdot \frac{|B|}{\left|2^{k} B\right|}
$$

$$
\left\|\nabla L^{-1 / 2}(a)\right\|_{L^{2}\left(2^{k+1} B \backslash 2^{k} B\right)} \leq C \cdot 2^{-k(2 N-n / p+n / 2)}\left|2^{k} B\right|^{1 / 2} w\left(2^{k} B\right)^{-1 / p} .
$$

Therefore, we have proved $\nabla L^{-1 / 2} a$ is a $w$ - $(p, 2,0,2 N-n / p+n / 2)$-molecule. This concludes the proof of Theorem 1.1. 


\section{References}

[1] P. Auscher, X. T. Duong, A. McIntosh, Boundedness of Banach space valued singular integral operators and Hardy spaces, preprint, 2004.

[2] E. B. Davies, Heat Kernels and Spectral Theory, Cambridge Univ. Press, 1989.

[3] X. T. Duong, E. M. Ouhabaz and L. X. Yan, Endpoint estimates for Riesz transforms of magnetic Schrödinger operators, Ark. Mat, 44(2006), 261-275.

[4] J. Dziubański and J. Zienkiewicz, Hardy space $H^{1}$ associated to Schrödinger operator with potential satisfying reverse Hölder inequality, Rev. Mat. Iberoamericana, 15(1999), 279-296.

[5] J. Dziubański and J. Zienkiewicz, $H^{p}$ spaces for Schrödinger operators, Fourier Analysis and Related Topics, Vol 56, 2002, 45-53.

[6] J. Dziubański and J. Zienkiewicz, $H^{p}$ spaces associated with Schrödinger operators with potentials from reverse Hölder classes, Colloq. Math, 98(2003), 5-38.

[7] J. Garcia-Cuerva, Weighted $H^{p}$ spaces, Dissertations Math, 162(1979), $1-63$.

[8] J. Garcia-Cuerva and J. Rubio de Francia, Weighted Norm Inequalities and Related Topics, North-Holland, Amsterdam, 1985.

[9] R. F. Gundy and R. L. Wheeden, Weighted integral inequalities for nontangential maximal function, Lusin area integral, and Walsh-Paley series, Studia Math, 49(1974), 107-124.

[10] S. Hofmann, G. Z. Lu, D. Mitrea, M. Mitrea, L. X. Yan, Hardy spaces associated to non-negative self-adjoint operators satisfying DaviesGaffney estimates, preprint, 2007.

[11] T. Kato, Perturbation Theory for Linear Operators, Second Edition, Springer-Verlag, Berlin, 1984.

[12] M. Y. Lee and C. C. Lin, The molecular characterization of weighted Hardy spaces, J. Funct. Anal, 188(2002), 442-460.

[13] B. Muckenhoupt, Weighted norm inequalities for the Hardy maximal function, Trans. Amer. Math. Soc, 165(1972), 207-226. 
[14] E. M. Ouhabaz, Analysis of Heat Equations on Domains, London Math. Soc. Monographs, Vol 31, Princeton Univ. Press, Princeton, NJ, 2005.

[15] Z. Shen, $L^{p}$ estimates for Schrödinger operators with certain potentials, Ann. Inst. Fourier(Grenoble), 45(1995), 513-546.

[16] A. Sikora, Riesz transform, Gaussian bounds and the method of wave equation, Math. Z, 247(2004), 643-662.

[17] B. Simon, Maximal and minimal Schrödinger forms, J. Operator Theory, 1(1979), 37-47.

[18] L. Song and L. X. Yan, Riesz transforms associated to Schrödinger operators on weighted Hardy spaces, J. Funct. Anal, 259(2010), 14661490.

[19] J. O. Stömberg and A. Torchinsky, Weighted Hardy spaces, Lecture Notes in Math, Vol 1381, Springer-Verlag, 1989. 